\title{
Microbial modulation of host apoptosis and pyroptosis
}

\author{
Yongqun $\mathrm{He}^{1 *}$ and Amal O. Amer ${ }^{2 *}$ \\ 1 Unit for Laboratory Animal Medicine, Department of Microbiology and Immunology, Center for Computational Medicine and Bioinformatics, Comprehensive \\ Cancer Center, University of Michigan Medical School, Ann Arbor, MI, USA \\ 2 Department of Microbial Infection and Immunity, Department of Internal Medicine, Center for Microbial Interface Biology, Ohio State University, Columbus, OH, \\ USA \\ *Correspondence: yongqunh@med.umich.edu; amal.amer@osumc.edu
}

Edited and reviewed by:

Yousef Abu Kwaik, University of Louisville School of Medicine, USA

Keywords: apoptosis, pyroptosis, microbial infection, caspase, inflammasome, Brucella, Legionella pneumophila, Mycobacterium tuberculosis

Apoptosis and pyroptosis are two common programmed cell death types induced by various microbial infections. Apoptosis is non-inflammatory programmed cell death and can be triggered through intrinsic or extrinsic pathways and with or without the contribution of mitochondria. Pyroptosis is an inflammatory cell death and is typically triggered by caspase- 1 after its activation by various inflammasomes. Non-canonical caspase-11-mediated pyroptosis has been identified. A NLRP3 (cryopyrin)-dependent but casepase-1-independent proinflammatory necrosis called pyronecrosis (Willingham et al., 2007), and a caspase-2dependent but caspase-1-independent proinflammatory cell death (Chen et al., 2011) have also been reported. Microbial pathogens are able to modulate host apoptosis, pyroptosis, and inflammasomes through different triggers and pathways. The promotion and inhibition of host cell death vary and depend on the microbe types, virulence, and phenotypes.

In this Special Research Topics issue, recent advances in microbial modulation of host programmed cell death, with a special focus on apoptosis and pyroptosis, were captured in a total of 11 research and review articles. The special issue includes three Original Research Articles, five Review Articles, and three Mini Review Articles. Two articles were published for each of the three pathogens: Brucella spp. (Bronner et al., 2013; Pei et al., 2014), Legionella pneumophila (Abu Khweek et al., 2013; Casson and Shin, 2013), and Mycobacterium tuberculosis (Aguilo et al., 2013; Parandhaman and Narayanan, 2014). Modulation of host immune defenses by Aeromonas and Yersinia species is introduced in Rosenzweig and Chopra (2013). While (Cunha and Zamboni, 2013) summarizes the subversion of inflammasome activation and pyroptosis by eight pathogenic bacteria, (Malireddi and Kanneganti, 2013) introduces the role of type I interferons in inflammasome activation and cell death induced by microbial infections. The apoptosis-associated uncoupling of bone formation and resorption in osteomyelitis is reviewed in Marriott (2013). The intestinal epithelial cell apoptosis in the setting of altered microbiota with enteral nutrient deprivation is reviewed in Demehri et al. (2013).

Brucella causes brucellosis, one of the most common zoonotic diseases in the world in humans and a variety of animal species. The Brucella-macrophage interaction is critical to Brucella virulence. Virulent smooth Brucella strains inhibit macrophage cell death. This is an important strategy employed by several intracellular pathogens to maintain the survival of the eukaryotic cell that represents its niche. Many attenuated rough Brucella strains induce macrophage cell death. The Original Research Article (Pei et al., 2014) demonstrates that after smooth Brucella invade and replicate inside host macrophages, some smooth bacteria can automatically dissociate into rough mutants that can then cause the macrophage cytotoxicity. The cytotoxicity of infected macrophages is critical for Brucella egress and dissemination. The macrophage necrotic cell death also induces inflammatory responses and recruits more macrophages to the infection site.

The rough attenuated $B$. abortus vaccine strain RB51 was found to induce caspase-2-mediated but caspase-1-independent apoptotic and necrotic cell death (Chen and He, 2009). Original Research Article (Bronner et al., 2013) from this special issue further illustrates this mechanism. In RB51-infected macrophages, caspase- 2 regulates many genes and several cell death pathways: (i) proapoptotic caspases- 3 and -8 activation; (ii) mitochondrial cytochrome $c$ release and TNF $\alpha$ production; (iii) caspase- 1 and IL-1 $\beta$ production driven by caspase-2-mediated mitochondrial dysfunction. Unlike S. typhimurium-induced caspase-1-mediated pyroptosis, RB51-induced pore formation does not contribute to RB51-induced proinflammatory cell death. Therefore, caspase-2 appears to act as a "master regulator" that regulates various genes and pathways and induces a hybrid cell death with features of both apoptosis and pyroptosis. The caspase-2-mediated cell death was also conserved in macrophages treated with cellular stress inducers including etoposide, naphthalene, or anti-Fas (Bronner et al., 2013).

Interesting study by $\mathrm{Abu}$ Khweek compared the innate immune response of planktonic and biofilm-derived L. pneumophila. L. pneumophila, the causative agent of Legionnaire's disease, replicates inside macrophages to establish infection. In the Original Research Article (Abu Khweek et al., 2013), the authors demonstrated that compared to planktonic L. pneumophila, biofilm-derived L. pneumophila (i) replicate more in murine macrophages, (ii) lacks flagellin expression, (iii) do not activate caspase-1 or -7 , (iv) trigger less cell death, and (v) are mostly enclosed in vacuoles that do not fuse with lysosomes. Therefore, biofilm-derived L. pneumophila which closely reproduces the natural mode of the bacterial infection in 
human is able to evade the innate immune response in murine macrophages.

The canonical pyroptosis is triggered by the inflammasome, a multi-protein complex assembled in the cytosol to activate caspase-1. A non-canonical inflammasome activates caspase-11 and also leads to pro-inflammatory cell death (Kayagaki et al., 2011). Independently of the inflammasome, caspase-11 promotes the fusion of the L. pneumophila-containing vacuole with the lysosome (Akhter et al., 2012). The diverse roles of caspase-11 and routes of activation are described in the mini-review (Casson and Shin, 2013) L. pneumophila triggers canonical caspase-1dependent inflammasome activation through one of two pathways: (i) Type 4 secretion system (T4SS)-regulated flagellin, NAIP5, and NLRC4; (ii) host ASC and NLRP3, and a L. pneumophila-derived unknown signal. Molecular details on caspase11 activation in L. pneumophila-infected macrophages remain unclear. Interestingly, the inflammasome pathway appears to cross talk with and autophagy, another immune response (Casson and Shin, 2013).

Mycobacterium tuberculosis, another professional intracellular pathogen in this issue, also manipulates cell death. Conflicting results have been reported to support inhibition or induction of apoptosis as a virulence mechanism employed by mycobacteria. This elegant review article (Aguilo et al., 2013), summarizes the evidences showing that ESX-1-induced apoptosis during mycobacterial infection contributes to bacterial virulence. The ESX-1 secretion system regulates the exportation of ESAT-6, a major virulence factor whose secretion is essential for M. tuberculosis-induced apoptosis. ESAT-6 appears to trigger the mitochondrial apoptotic pathway through ER-stress activation. ESX-1 dependent apoptosis supports cell-to-cell colonization and bacterial spread. Highly apoptogenic M. tuberculosis nuoG mutant showed higher cell-to-cell spread and increased antigen crosspresentation favoring the host. It is evident that apoptosis may benefit the host or mycobacterial pathogen according to different experimental conditions (Aguilo et al., 2013).

The well rounded report (Parandhaman and Narayanan, 2014) summarizes more than 10 different cell death modalities involving M. tuberculosis. The paper also reviews how $\mathrm{PknE}$, one of 11 mycobacterial serine/threonine protein kinases, inhibits apoptosis and benefits the bacterial survival.

This issue also comprises a paper (Rosenzweig and Chopra, 2013) that describes toxins secreted by pathogenic Yersiniae and most Aeromonas species that modulate infected host cell death. The T3SS effector Yersinia outer membrane protein J (YopJ) is an acetyltransferase that disrupts MAPK and NF- $\kappa B$ signaling pathways to favor apoptosis and pyroptosis induction. Similarly, Aeromonas hydrophila AexU protein induces apoptosis by targeting NF- $\mathrm{B}$ signaling. Additionally Aeromonas includes T2- and T6SS effectors that further modulate host immune responses to promote bacterial virulence (Rosenzweig and Chopra, 2013).

The nice paper by Zamboni's group (Cunha and Zamboni, 2013) first reviews different types of inflammasomes that activate caspase-1 (via NLRC4, AIM2, or NLRP3) or caspase-11 and then lead to pyroptosis. These host inflammasomes and pyroptosis pathways can be targeted by microbial factors released via T3SS/T4SS or other mechanisms in different pathogens. This paper reviews the mechanisms employed by eight bacterial species to evade inflammasome activation and pyroptosis induction. These bacteria include Chlamydia trachomatis, Coxiella burnetii, Francisella tularensis, Legionella pneumophila, Pseudomonas aeruginosa, Shigella flexneri, Vibrio parahaemolyticus, and Yersinia spp. (Cunha and Zamboni, 2013).

On the host side, the review by Kanneganti's group (Malireddi and Kanneganti, 2013) in this issue introduces the role of type I interferons in inflammasome activation and cell death during infections of five intracellular, four extracellular bacteria, viruses, and fungi.

How cell death can lead to human disease conditions is well described by the review paper (Marriott, 2013) that focuses on osteomyelitis, a severe infection of bone caused by $S$. aureus and Salmonella spp. Osteomyelitis is often associated with bone resorption and progressive inflammatory destruction. In the paper Marriott describes the mechanisms underlying the destruction of bone tissue, with a focus on the apoptosis-associated uncoupling of bone formation and resorption in osteomyelitis. Different microbial virulence factors, host response genes and pathways, and their interactions during the formation of osteomyelitis are introduced.

It has been well established that pathogens modulate apoptosis and pyroptosis, but what about microbiota? The paper (Demehri et al., 2013) in this issue introduces a shift in our understanding of intestinal microbiota such as Gram-negative Proteobacteria after enteral nutrient deprivation. The altered microbiota setting leads to increased intestinal proinflammatory cytokines, decreased epithelial cell proliferation, and increased epithelial cell apoptosis. These eventually cause the loss of epithelial barrier function.

The cover image of this E-book summarizes the key findings reported in the original research, review, or mini-review articles included in this e-book.

As briefly introduced above, this special Research Topic issue covers a broad range of cases and reviews demonstrating the modulation of host cell death pathways by different bacterial pathogens and resident microbiota. While huge progress has been made in the past decades, many challenging questions still remain.

\section{REFERENCES}

Abu Khweek, A., Fernandez Davila, N. S., Caution, K., Akhter, A., Abdulrahman, B. A., Tazi, M., et al. (2013). Biofilm-derived Legionella pneumophila evades the innate immune response in macrophages. Front. Cell. Infect. Microbiol. 3:18. doi: $10.3389 /$ fcimb. 2013.00018

Aguilo, N., Marinova, D., Martin, C., and Pardo, J. (2013). ESX-1-induced apoptosis during mycobacterial infection: to be or not to be, that is the question. Front. Cell. Infect. Microbiol. 3:88. doi: 10.3389/fcimb.2013.00088

Akhter, A., Caution, K., Abu Khweek, A., Tazi, M., Abdulrahman, B. A., Abdelaziz, D. H., et al. (2012). Caspase-11 promotes the fusion of phagosomes harboring pathogenic bacteria with lysosomes by modulating actin polymerization. Immunity 37, 35-47. doi: 10.1016/j.immuni.2012.05.001

Bronner, D., O’Riordan, M., and He, Y. (2013). Caspase-2 mediates a Brucella abortus RB51-induced hybrid cell death having features of apoptosis and pyroptosis. Front. Cell. Infect. Microbiol. 3:38. doi: 10.3389/ fcimb.2013.00083

Casson, C. N., and Shin, S. (2013). Inflammasome-mediated cell death in response to bacterial pathogens that access the host cell cytosol: lessons from legionella pneumophila. Front. Cell. Infect. Microbiol. 3:111. doi: 10.3389/fcimb.2013.00111 
Chen, F., Ding, X., Ding, Y., Xiang, Z., Li, X., Ghosh, D., et al. (2011). Proinflammatory caspase-2-mediated macrophage cell death induced by a rough attenuated Brucella suis strain. Infect. Immun. 79, 2460-2469. doi: 10.1128/IAI.00050-11

Chen, F., and He, Y. (2009). Caspase-2 mediated apoptotic and necrotic murine macrophage cell death induced by rough Brucella abortus. PLoS ONE 4:e6830. doi: 10.1371/journal.pone.0006830

Cunha, L. D., and Zamboni, D. S. (2013). Subversion of inflammasome activation and pyroptosis by pathogenic bacteria. Front. Cell. Infect. Microbiol. 3:76. doi: 10.3389/fcimb.2013.00076

Demehri, F. R., Barrett, M., Ralls, M. W., Miyasaka, E. A., Feng, Y., and Teitelbaum, D. H. (2013). Intestinal epithelial cell apoptosis and loss of barrier function in the setting of altered microbiota with enteral nutrient deprivation. Front. Cell. Infect. Microbiol. 3:105. doi: 10.3389/fcimb. 2013.00105

Kayagaki, N., Warming, S., Lamkanfi, M., Vande Walle, L., Louie, S., Dong, J., et al. (2011). Non-canonical inflammasome activation targets caspase-11. Nature 479, 117-121. doi: 10.1038/nature10558

Malireddi, R. K., and Kanneganti, T. D. (2013). Role of type I interferons in inflammasome activation, cell death, and disease during microbial infection. Front. Cell. Infect. Microbiol. 3:77. doi: 10.3389/fcimb.2013.00077

Marriott, I. (2013). Apoptosis-associated uncoupling of bone formation and resorption in osteomyelitis. Front. Cell. Infect. Microbiol. 3:101. doi: 10.3389/fcimb.2013.00101

Parandhaman, D. K., and Narayanan, S. (2014). Cell death paradigms in the pathogenesis of Mycobacterium tuberculosis infection. Front. Cell. Infect. Microbiol. 4:31. doi: $10.3389 /$ fcimb.2014.00031
Pei, J., Kahl-McDonagh, M., and Ficht, T. A. (2014). Brucella dissociation is essential for macrophage egress and bacterial dissemination. Front. Cell. Infect. Microbiol. 4:23. doi: 10.3389/fcimb.2014.00023

Rosenzweig, J. A., and Chopra, A. K. (2013). Modulation of host immune defenses by Aeromonas and Yersinia species: convergence on toxins secreted by various secretion systems. Front. Cell. Infect. Microbiol. 3:70. doi: 10.3389/fcimb.2013.00070

Willingham, S. B., Bergstralh, D. T., O’Connor, W., Morrison, A. C., Taxman, D. J., Duncan, J. A., et al. (2007). Microbial pathogen-induced necrotic cell death mediated by the inflammasome components CIAS1/cryopyrin/NLRP3 and ASC. Cell Host Microbe 2, 147-159. doi: 10.1016/j.chom.2007.07.009

Conflict of Interest Statement: The authors declare that the research was conducted in the absence of any commercial or financial relationships that could be construed as a potential conflict of interest.

Received: 01 June 2014; accepted: 03 June 2014; published online: 19 June 2014.

Citation: He Y and Amer AO (2014) Microbial modulation of host apoptosis and pyroptosis. Front. Cell. Infect. Microbiol. 4:83. doi: 10.3389/fcimb.2014.00083

This article was submitted to the journal Frontiers in Cellular and Infection Microbiology.

Copyright (C) 2014 He and Amer. This is an open-access article distributed under the terms of the Creative Commons Attribution License (CC BY). The use, distribution or reproduction in other forums is permitted, provided the original author(s) or licensor are credited and that the original publication in this journal is cited, in accordance with accepted academic practice. No use, distribution or reproduction is permitted which does not comply with these terms. 